\title{
Focus on intracytoplasmic morphologically selected sperm injection (IMSI): a mini-review
}

\author{
Giuseppe Lo Monte ${ }^{1}$, Fabien Murisier ${ }^{2}$, Isabella Piva ${ }^{1}$, Marc Germond ${ }^{2}$ and Roberto Marci ${ }^{1}$
}

Intracytoplasmic sperm injection (ICSI) is the recommended treatment in many cases of male-factor infertility. Several studies have demonstrated a positive correlation between optimal sperm morphology and positive ICSI outcomes. In fact, spermatozoa with severe abnormalities of the head are well documented to be associated with low fertilisation, implantation and pregnancy rates. However, a spermatozoon which is classified as 'normal' by microscopic observation at low magnification could contain ultrastructural defects that impair both the fertilisation process and embryonic development. The intracytoplasmic morphologically selected sperm injection (IMSI) procedure changed the perception of how a spermatozoon suitable for injection should appear. Sperm selection is carried out at $\times 6000$ magnification, allowing improved assessment of the sperm nucleus. Currently, standardized clinical indications for IMSI are lacking and the candidates are selected on the grounds of their medical history or of a careful analysis of the sperm suspension. Further prospective randomized studies are needed to confirm the advantages of IMSI in specific groups of patients. In addition to providing a brief overview of the IMSI procedure, this study aims to review the literature, which explains the theoretical basis and the clinical outcomes of this technique. Several reports show that IMSI is associated with improved implantation and clinical pregnancy rates as well as lower abortion rates when compared to ICSI. Although a possible correlation between the sperm's abnormal nucleus shape, increased DNA fragmentation and negative laboratory and clinical outcomes has been long investigated, the results are conflicting. Asian Journal of Andrology (2013) 15, 608-615; doi:10.1038/aja.2013.54; published online 8 July 2013

Keywords: assisted reproduction technologies; ICSI; IMSI; infertility

\section{INTRODUCTION}

Approximately $10 \%$ of couples are estimated to seek assistance for infertility problems during their reproductive years. According to the American Society of Reproductive Medicine, one-third of couples' infertility is due to a male factor and another third should be attributed to female-related problems. In the remaining third of the couples, the cause of infertility remains unexplained or is related to a combination of male and female factors. ${ }^{1}$ The underlying conditions can be identified and treated only in a few cases and thus assisted reproductive technologies are often considered as the first-line treatment to achieve pregnancy in infertile couples.

Assisted reproductive technology increases the pregnancy probability by a double mechanism; first, it facilitates the interaction between spermatozoa and oocytes, and second, it bypasses seminal abnormalities, such as a reduced number, motility or increased morphological defects of spermatozoa. Sperm morphology was suggested to play an important role in determining fertility and was proven to be closely related to fertilisation and pregnancy rates in the natural fertilisation process $^{2,3}$ as well as in intrauterine insemination and in conventional in vitro fertilisation (IVF) treatments. ${ }^{4}$ Normal sperm morphology is necessary for their successful passage through the zona pellucida and fusion with the plasma membrane of the oocyte. In fact, it is well documented that morphological abnormalities of the sperm head (round, large or tapered) are associated with low fertilisation, implantation and pregnancy rates. ${ }^{5-7}$ Because the intracytoplasmic sperm injection (ICSI) procedure involves the direct injection of the spermatozoon into the oocyte, embryologists considered that the morphological evaluation of male gametes was of secondary importance. $^{8}$

Several studies have demonstrated a positive correlation between sperm morphology and positive ICSI outcomes. ${ }^{9,10}$ However, the overall appearance of a sperm sample would not always reflect the quality of the single spermatozoon selected for the microinjection procedure. A spermatozoon classified as 'normal' using low power microscopic observations may carry ultrastructural defects that impair the fertilisation process and embryonic development. Severe oligozoospermia was shown to be frequently associated with structural and numerical chromosomal alterations. ${ }^{11,12}$ Additionally, this condition is often linked with lower fertilisation and live birth rates as well as with a higher probability of phenotypic abnormalities and chromosomal aberrations in the offspring. ${ }^{13,14}$ Moreover, several studies have clarified the association between a high rate of sperm nuclear DNA fragmentation, altered sperm nuclear morphology and poor embryo quality. ${ }^{15,16}$ The use of tested gametes for reproductive purposes is not possible because all the available tests for functional and genetic spermatozoa evaluation have proven to be highly cytotoxic. Thus, several non-invasive techniques have been recently introduced to increase the ICSI success rates. 
In 2002, Bartoov et al. developed a new method for the evaluation of human spermatozoa called motile sperm organelle morphology examination (MSOME) ${ }^{17}$ This device allows the observation of spermatozoa at high magnification $(>\times 6000)$ by using an inverted light microscope. The application of MSOME for conventional IVF or ICSI candidates has led to the development of intracytoplasmic morphologically selected sperm injection (IMSI). ${ }^{18}$ Currently, IMSI is the only real-time method that involves unstained sperm cells and hence enables the detection of spermatozoa with ultrastructural defects to be discarded. Therefore, IMSI could be considered as an 'advanced ICSI'.

The aim of this study is to provide a brief overview of the current understanding of IMSI, as well as the theoretical basis underlying its use and of the clinical outcomes achieved by this technique.

\section{ICSI: key issues and unsolved problems}

Currently, ICSI is the most efficient technique in terms of oocyte fertilisation in IVF programs. For many years, sperm motility has been considered as the most important factor in determining ICSI outcomes. ${ }^{19}$ Conversely, the importance of sperm morphology has been widely debated. de Vos et al. ${ }^{9}$ evaluated the impact of individual sperm morphology on ICSI outcomes, including fertilisation rate, embryo development and implantation rate, in 568 cycles. Most of the injected spermatozoa from ejaculated samples $(91.3 \%)$ presented an apparently normal morphology, whilst the remaining $8.7 \%$ showed an amorphous head in the majority of the cases. Lower percentages of normal-shaped sperm cells were observed in surgically retrieved samples. The use of spermatozoa extracted from semen samples with an apparently normal sperm morphology resulted in higher fertilisation, implantation and pregnancy rates compared with the injection of an abnormal spermatozoon $(71.7 \%$ vs. $60.7 \%, 20.2 \%$ vs. $36.7 \%$ and $9.6 \%$ vs. $18.7 \%$, respectively). The low magnification $(\times 400)$ and resolution of the microscope used for the morphology assessment represented the main limitation of this study.

Several studies have reported that altered sperm morphology, and specifically the head abnormalities, may correspond to hidden chromosomal defects, especially those involving chromatin packaging. ${ }^{11,20}$ Both DNA fragmentation and unsuccessful sperm chromatin decondensation might be responsible for fertilisation failure. ${ }^{21,22}$ Larson et al. ${ }^{23}$ reported poor IVF outcomes when using semen samples that contained over $27 \%$ spermatozoa with substantial DNA denaturation. More specifically, the pregnancy rate associated with semen samples containing over $27 \%$ spermatozoa with substantial DNA denaturation was $0 \%$, while the rate reached $24 \%$ in the case of semen samples containing under $27 \%$ spermatozoa with substantial DNA denaturation. Therefore, one could speculate that although chromatin abnormalities may not affect the fertilisation rate, they can influence the subsequent embryonic development and hence reduce the clinical pregnancy rates. On the basis of these observations, such chromatin alterations in spermatozoa have been suggested to cause repeated ICSI failures.

Several authors have investigated whether ICSI could negatively affect the health of the offspring by facilitating congenital malformations and childhood cancers or by compromising the offspring's future fertility. The theoretical risk of this procedure is related to the micro-injection of spermatozoa that could potentially carry hidden genetic alterations. ${ }^{24-26}$

In 2005, a multicentre cohort study ${ }^{25}$ assessed the physical health of children who were either naturally conceived $(n=538)$ or conceived after ICSI $(n=540)$ or after conventional IVF $(n=437)$. Children born after ICSI and IVF were demonstrated to require more healthcare resources than those who were conceived naturally. The odds ratio of a major malformation was 2.77 (95\% CI: 1.41-5.46) for children born after ICSI and 1.80 (95\% CI: 0.85-3.81) for children born after IVF, compared with the naturally conceived group. Additionally, a higher prevalence of male urogenital malformations was observed. However, recent studies have shown that the risk of malformations could be related to male infertility per se regardless of the type of IVF treatment. ${ }^{24}$

Further concerns have been raised about the possible transmission of subtle parental genetic alterations that could affect the offspring's future fertility. ${ }^{26}$

\section{IMSI: An improved ICSI?}

MSOME was first applied to the leftover sperm fraction selected for microinjection in 100 couples opting for ICSI treatment. ${ }^{17}$ MSOME was applied exclusively to the motile spermatozoa fraction. Moreover, sperm cells with severe malformations that were already evident at low magnification were excluded because they are not routinely used for microinjection. Each sperm cell was evaluated according to the morphological status of six subcellular organelles comprising the acrosome, post-acrosomal lamina, neck, mitochondria, tail and nucleus. These subcellular organelles were considered as morphologically abnormal on the basis of specific malformations as shown in Figure 1. Only $33 \%$ of spermatozoa from the examined samples appeared morphologically normal according to MSOME criteria, and a positive correlation was found between their morphological normality and the fertilisation rate. Nuclear normality (the shape of the nucleus and the presence of one or more vacuoles occupying more than $4 \%$ of the nuclear area) appeared to be the most important parameter to influence fertilisation and pregnancy rates. None of the couples who exhibited less than $20 \%$ spermatozoa with a morphologically normal nucleus achieved pregnancy. Other morphological characteristics of spermatozoa (acrosome, post-acrosomal lamina, neck, mitochondria and tail) examined in this study did not influence the final ICSI outcome.

The new MSOME approach was subsequently adopted for the sperm selection process that precedes the micro-injection. This new IVF procedure, called intracytoplasmic morphologically selected sperm injection (IMSI) by its authors, ${ }^{18}$ was performed on 62 couples with at least two previous failed ICSI cycles. These patients were compared to a control group comprising 50 couples undergoing conventional ICSI treatments. The aim of this study was to identify the best candidate spermatozoon for micro-injections based on the strict and specific morphological MSOME criteria. The IMSI approach resulted in significantly higher implantation and pregnancy rates than those achieved using conventional ICSI $(27 \%$ vs. $9.5 \%$ and $66 \%$ vs. $30 \%$, respectively) and in a considerably lower abortion rate (9\% vs. $33 \%)$.

\section{IMSI technique}

An inverted light microscope, equipped with high-power differential interference contrast optics enhanced by digital imaging, achieves a magnification of up to $\times 6600$ and allows the real-time assessment of morphological characteristics of motile spermatozoa (Figure 2). The specific features of the microscopes used in each study are variable, although the amplification system proposed by Bartoov et al. ${ }^{17}$ is the most widely applied. The inverted microscope is equipped with the Nomarski differential interference contrast optics, an Uplan Apo $\times 100$ oil/1.50 objective lens and a 0.55 numerical aperture condenser lens. The images are captured by a colour video camera containing a 0.5 inch, three-chip power HAD CCD (total calculated magnification 


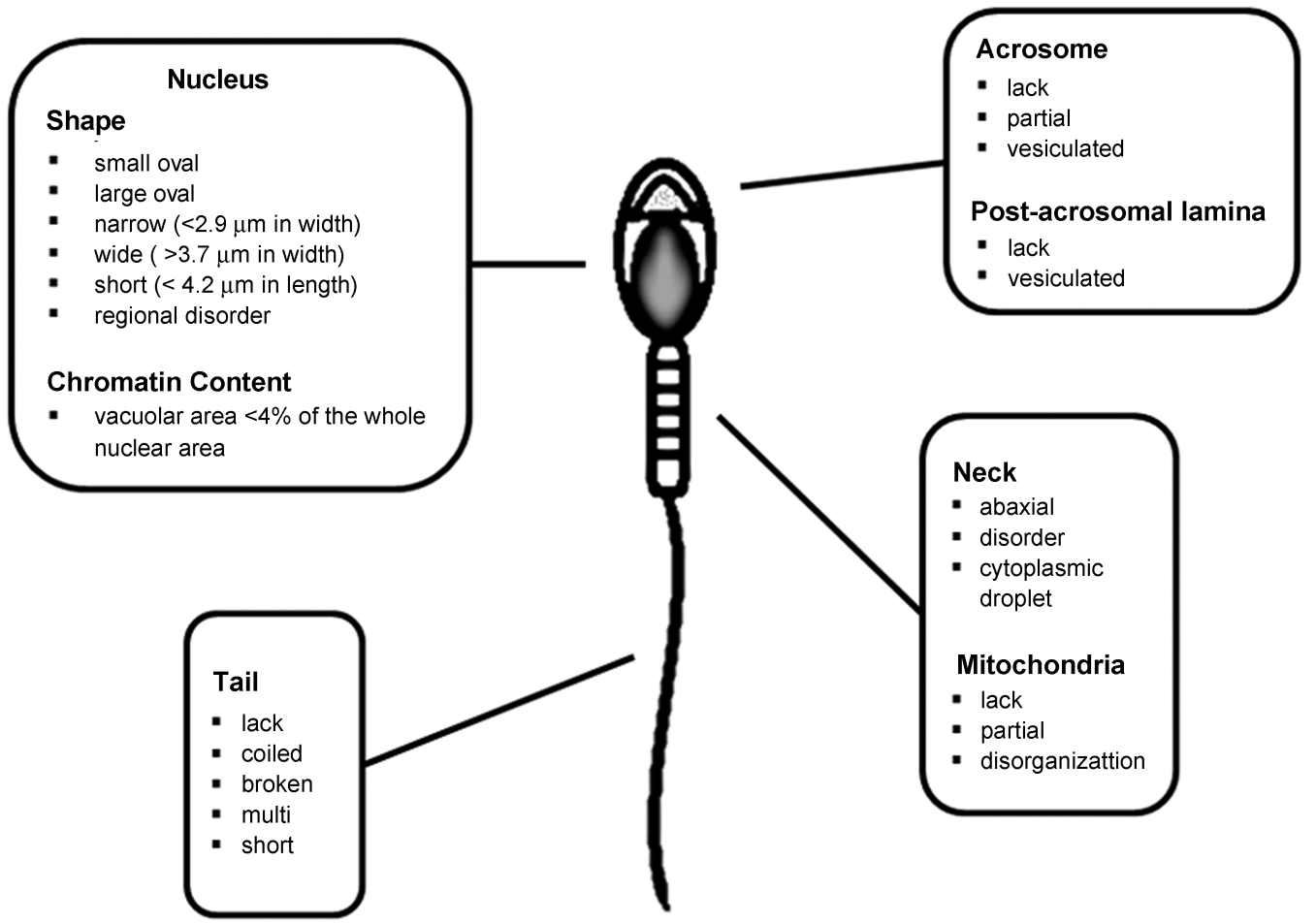

Figure 1 Sperm morphological features according to MSOME criteria. MSOME, motile sperm organelle morphology examination.

$\times 6600)$ and visualized on a monitor screen with diagonal dimension of $355.6 \mathrm{~mm}$. The selection of spermatozoa is often performed on glass-bottomed dishes to achieve the best optical quality, whereas microinjections are performed on a separate plastic dish. Semen sample preparation requires a specific procedure that varies according to the semen quality. The selection phase requires a suspension of spermatozoa prepared from discontinuous density gradient centrifugation. The pellet is then suspended in SPERM culture medium (Medi-Cult, Jyllinge, Denmark) to obtain a final concentration of motile sperm cells of approximately four million spermatozoa per millilitre. In patients affected by severe oligozoospermia $(<$ one million sperm cells per ejaculate) a low-density layer is preferably used and the pellet is subsequently suspended in $0.1-0.2 \mathrm{ml} \mathrm{sperm} \mathrm{me-}$ dium. ${ }^{18}$ The sperm suspension is then deposited in polyvinylpyrrolidone microdroplets covered with mineral oil. Polyvinylpyrrolidone is a viscous medium used to reduce sperm cells' motility to facilitate their morphological evaluation. The sample temperature and polyvinylpyrrolidone concentration depend on sperm motility. The morphologically normal spermatozoon is then aspirated with an ICSI injection pipette and subsequently added to a microdroplet of medium on the plastic Petri dish. The droplet is then covered with mineral oil and the injection is finally performed.

Because the detailed analysis of each sperm takes longer than the conventional approaches, the IMSI procedure lasts roughly twice as long as regular ICSI. This emphasizes the requirement for a sufficient time period within the routine laboratory schedule.

\section{Selection criteria}

The MSOME selection criteria are based on an arbitrary descriptive approach taken from different studies that assess sperm morphology through transmission electron microscopy and scanning electron microscopy. ${ }^{27,28}$ The microscopic examination of each sperm sample involves the evaluation of six subcellular organelles: (i) acrosome; (ii) post-acrosomal lamina; (iii) neck; (iv) mitochondria; (v) principal piece of the tail (or flagellum); and (vi) nucleus (Figure 1). With regard to the sperm head, three parameters should be taken into account: the shape, the presence of vacuoles and the base. Among all the subcellular organelles, the normal morphology of the sperm cell

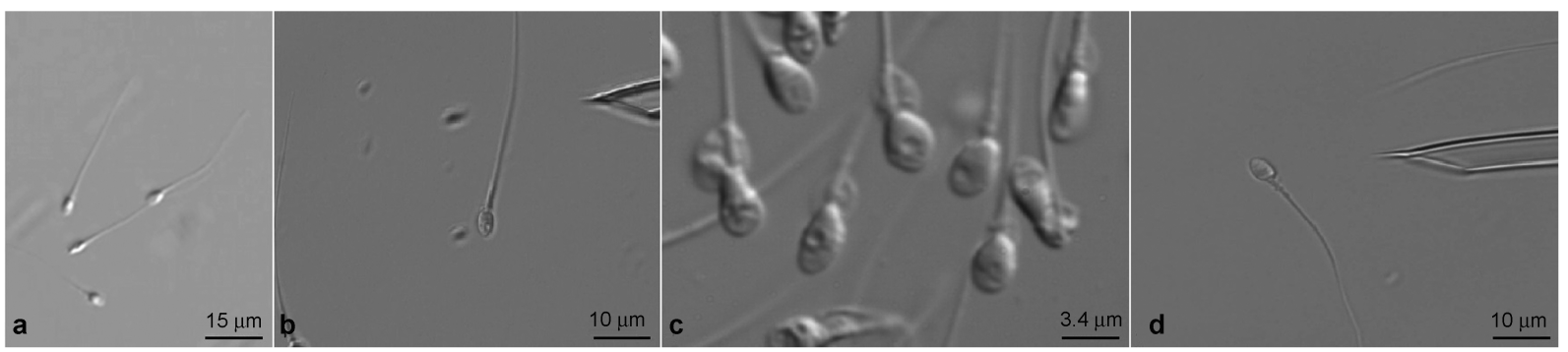

Figure 2 Spermatozoa observed by low magnification (a) and high magnification (b-d). The shape and presence of vacuoles can be clearly observed in c. A vacuolefree spermatozoon is shown in $\mathbf{d}$ 
nucleus may be the crucial characteristic associated with a positive ICSI outcome. Specifically, the morphological appearance of the sperm nucleus is assessed according to both the shape and to the chromatin mass appearance. A normally shaped sperm nucleus is usually smooth, symmetrical and oval and displays a homogeneous chromatin mass without extrusions or invaginations. Moreover, the nucleus does not demonstrate more than one vacuole involving less than $4 \%$ of the nuclear area. A normal head base is U-shaped. The acrosome and post-acrosomal lamina are considered abnormal if absent, partial or vesiculated. An abaxial deviation and the presence of cytoplasmic droplets or other disorders account for neck abnormalities. A normal spermatozoon should not present with broken, short or double and coiled tails either. Mitochondrial sperm abnormalities include observations where they are partially formed, disorganized, or are absent.

\section{Indications for IMSI: a theoretical basis}

The indications for IMSI are frequently indirect, such as recurrent implantation failure, high levels of DNA fragmentation, high levels of sperm aneuploidy or repeated early abortions. ${ }^{18,29,30}$

Several reports suggest that damaged sperm DNA adversely affects reproductive outcomes and that infertile men present substantially more DNA-damaged sperm than fertile men. ${ }^{31,32}$ In an attempt to evaluate the role of high-magnification selection for couples with a high degree of sperm DNA fragmentation, Hammoud et al. ${ }^{33}$ analysed the DNA integrity in six types of spermatozoa. The six categories included the following: (i) motile spermatozoa selected at $\times 200$ magnification; (ii) motile and morphologically normal spermatozoa selected at $\times 200$ magnification; (iii) motile spermatozoa with vacuoles located in the anterior half of the head selected at $\times 6300$ magnification; (iv) motile spermatozoa with vacuoles located in the posterior half of the head selected at $\times 6300$ magnification; (v) motile spermatozoa without vacuoles selected at $\times 6300$ magnification; and (vi) non-selected spermatozoa. After the selection process, spermatozoa were fixed and subsequently evaluated for DNA fragmentation using the terminal deoxynucleotidyl transferase dUTP nick-end labelling assay. Motile normal spermatozoa with a vacuole-free head selected at $\times 6300$ magnification showed a significantly lower sperm DNA fragmentation rate $(4.1 \% \pm 1.1 \%)$ than all other types of spermatozoa (type a: $20.8 \% \pm 2.7 \%$; type b: $18.7 \% \pm 2.7 \%$; type c: $15.9 \% \pm 2.9 \%$; and type d: $22.5 \% \pm 3.6 \%)$. Conversely, non-selected spermatozoa demonstrated the highest DNA fragmentation rate $(26.1 \% \pm 1.5 \%)$. Thus, the selection of normal spermatozoa with non-fragmented DNA in patients with high sperm DNA fragmentation rates should be performed at $\times 6300$ magnification, which appears to be more effective than using a $\times 200$ magnification.

The macrocephalic sperm head syndrome is closely associated with abnormal sperm chromatin content, abnormally shaped heads and multiple flagella. Chelli et al. ${ }^{34}$ recently published a study that assessed the chromosomal content of IMSI-selected spermatozoa in two patients affected by this syndrome. Although IMSI allowed the elimination of polyploid spermatozoa, it did not allow the identification of euploid spermatozoa. Thus, the authors suggested that the selection of normal head shape spermatozoa is not sufficient to guarantee normal chromosomal content. ${ }^{34}$

To date, the origin of the nuclear vacuoles and their role in the early phases of embryo development are still unclear. Hazout et al. ${ }^{29}$ studied the association between nuclear shape normality and the clinical and biological outcome of ICSI in 125 couples with at least two previous ICSI failures. All couples underwent a further conventional ICSI attempt followed by an IMSI attempt. The clinical outcome was significantly different after the two treatments and showed increased pregnancy $(37.6 \%$ vs. $2.4 \%)$, implantation $(20.3 \%$ vs. $0.8 \%)$, delivery $(33.6 \%$ vs. $0 \%)$ and birth $(17.6 \%$ vs. $0 \%)$ rates in the IMSI group. This favourable clinical impact was not accompanied by a parallel improvement in the biological outcome in terms of the fertilisation rate, embryo cleavage rate and the embryo morphology grades. Sperm DNA fragmentation was subsequently assessed using the DNA terminal deoxynucleotidyl transferase dUTP nick-end labelling assay in 72 of the couples enrolled and proved not to be associated with the positive clinical outcome observed after IMSI procedure. Thus, this evidence does not support a correlation between the presence of large nuclear vacuoles and sperm DNA fragmentation on the one hand and negative results on the other.

Moreover, Kacem et al. ${ }^{35}$ demonstrated that these vacuoles are mostly located in the anterior sperm head region, thus supporting a possible relationship with the acrosome rather than with a potential chromatin defect. In the aforementioned study, the evaluation of both the sperm morphology and the acrosomal status were simultaneously performed on fixed spermatozoa. Sperm morphology was detected at a high magnification using the same optics used for MSOME, while the acrosome status was assessed using agglutinin-fluorescein isothiocyanate staining and epifluorescence. Sixty-one percent of spermatozoa that presented with large vacuoles showed green staining in the anterior region of the sperm head and corresponded to the acrosome location. Moreover, the induction of the acrosome reaction (using ionophore A23587) increased the percentage of vacuole-free spermatozoa (from $41.2 \%$ to $63.8 \%$ ), suggesting that most of the nuclear vacuoles are of acrosome origin. The components of the acrosome, such as trypsin-like acrosin and hyaluronidase, may exert a toxic effect on embryo development ${ }^{36}$ and supports the view that the injection of vacuolated spermatozoa could impair ICSI outcomes.

Peer et al. ${ }^{37}$ reported a higher frequency of vacuolated nuclei after incubation of the sperm samples in culture media at $37{ }^{\circ} \mathrm{C}$ than at $21{ }^{\circ} \mathrm{C}$. This suggests that prolonged sperm manipulation (exceeding two hours) should be performed at $21^{\circ} \mathrm{C}$ instead of $37^{\circ} \mathrm{C}^{37}$ to prevent excessive vacuolation.

Currently, insufficient data are available about the role of sperm aneuploidy in infertility or assisted reproduction. A study by Shi and Martin ${ }^{38}$ reported a correlation between increased levels of chromosomal abnormalities in spermatozoa and impaired semen parameters, such as sperm concentration and morphology alterations, in infertile men with normal karyotypes. Alterations of seminal fluid parameters, including oligozoospermia, asthenozoospermia and teratozoospermia, appear to be associated with increased sperm aneuploidy. Clearly, the increased frequency of such abnormalities is closely connected with the severity of infertility, and the highest levels are reported in men affected by severe oligoasthenoteratozoospermia and in spermatozoa retrieved from testicular sperm extraction in cases of non-obstructive azoospermia. ${ }^{38-40}$

Figueira et al. ${ }^{40}$ used IMSI to evaluate the morphology of the injected sperm and performed a preliminary study to examine its effect on embryo development at the chromosomal level. The authors enrolled couples undergoing their first IVF treatment in conjunction with pre-implantation genetic screening for aneuploidy as a result of the advanced maternal age. The patients were randomly allocated to receive ICSI or IMSI for the sperm selection procedure. The percentage of embryos showing autosomal aneuploidy did not differ significantly between the groups ( $56.9 \%$ vs. $51.3 \%)$. However, a significantly higher incidence of sex chromosome aneuploidy was observed in 
ICSI-derived embryos (23.5\% vs. $15.0 \%)$. Spermatozoa without nuclear morphological malformations were significantly associated with a lower incidence of aneuploidy in the derived embryos, which resulted in lower cycle termination rates. ${ }^{40}$

The poor quality of spermatozoa can adversely affect embryonic development. Vanderzwalmen et al. ${ }^{41}$ and Cassuto et al. ${ }^{42}$ evaluated the effect of specific sperm defects detected at high magnification on embryo development. A sperm quality score for each study group was generated based on specific parameters such as the normality of the head, the presence and size of vacuoles and the symmetry of the sperm head base. In both studies, the authors reported a significant correlation between the presence of large vacuoles in the nuclei of spermatozoa and a considerable impairment of the blastocyst quality on day 5 .

The results provided by a comparative prospective randomized study by Knez et al. ${ }^{43}$ appear consistent with the aforementioned investigations. These authors compared IMSI to ICSI in couples who presented with male infertility that had all resulted in growtharrested embryos following a prolonged 5-day embryo culture. A higher number of blastocysts per cycle were detected in the IMSI group compared to the ICSI group ( 0.80 vs. 0.65$)$ after a prolonged 5-day embryo culture. Furthermore, the IMSI procedure was associated with a significantly lower number of cycles with all growth arrested embryos and facilitated a reduced number of cycles with no embryonic transfer $(0 \%$ vs. 27.0\%). Moreover, a tendency towards higher implantation and pregnancy rates per cycle was recorded in the IMSI group (Table 1). ${ }^{43}$

Knez et al. also investigated whether IMSI could improve the embryo quality in terms of pronuclear morphology, embryonic development and blastomeric viability in a selected group of patients affected by severe teratozoospermia. IMSI generated a higher clinical pregnancy rate than ICSI ( $48 \%$ vs. $24 \%)$. Moreover, the use of IMSI facilitated an increase in the number of morphologically normal zygotes and an increase in the blastocyst formation rate and reduced the proportion of growth-arrested embryos. Based on this evidence, the authors argued for IMSI as a method of choice for cases of severe teratozoospermia. ${ }^{44}$

The negative effect of sperm nuclear vacuoles on blastocyst development could be due to an early or late paternal influence on initial embryo development. Paternal DNA begins to contribute to embryo development approximately 3 days after fertilisation. A late paternal influence occurring after this period could result in embryonic developmental arrest or could cause early abortions. ${ }^{45}$ Spermatozoa can also impair early embryonic development through several mechanisms, including fertilisation failure, embryo fragmentation or embryonic development arrest at the second pronuclear stage. ${ }^{45}$ The analysis of embryo quality is commonly performed on days 2 and 3 , but a possible impairment of embryo quality detected after day 2 could account for a late paternal effect rather than an early effect. Therefore, in the study by Mauri et al., ${ }^{46}$ the analysis of embryo quality was limited to day 2 . The authors did not find a significant difference on day 2 in the rate of high-quality embryos between IMSI (52.2\%) and ICSI (57.8\%). Thus, high-magnification sperm selection has been suggested not to influence the early paternal effects. ${ }^{46}$

All published reports dealt with the relationship between sperm head morphology and IMSI outcome. However, at present, only one of these studies ${ }^{47}$ assessed the influence of the morphology of the sperm midpiece observed at high magnification, on ICSI outcomes. The authors of this work hypothesized that a morphologically abnormal sperm midpiece could be associated with an altered centrosomal function. During fertilisation, the centrosome derived from the spermatozoon organizes the radial array of microtubules, including the sperm aster. An aberrant microtubule organisation may be responsible for fertilisation failure owing to a sperm centrosomal dysfunction. A heterologous ICSI of human spermatozoa into bovine oocytes was used to assess human sperm centrosomal function and to analyse sperm aster formation. The samples were divided into two groups according to the morphological features of the sperm midpiece (straight or tapered). To evaluate the differences between the groups, the proximal and the distal diameters of the midpiece were measured. Spermatozoa with vacuoles within the head were excluded from the study. Because normal magnification microscopy does not allow the evaluation of the morphological features of the sperm midpiece, sperm selected using conventional microscopy $(\times 400)$ were defined as controls.

The aster formation rate was lower in the subset of spermatozoa with tapered midpieces than in the spermatozoa with morphologically straight midpieces and in the control group $(33.3 \%, 80.5 \%$ and $69.6 \%$, respectively). Furthermore, the authors investigated the ultrastructure of the sperm midpiece region using electron microscopy. Sperm samples with tapered midpieces presented with mitochondrial misalignment and vacuolar structures around the midpieces, but no differences in the centrosomal structure were reported. As shown by this study, the evaluation of the midpiece morphology using high-magnification microscopy might improve the sperm aster formation rate. In our opinion, the misalignments of the organelles could be considered a morphological sign of sperm centrosomal dysfunction. However, to the best of our knowledge, this hypothesis is not supported by any other evidence. Thus, the possible mechanism by which this abnormal midpiece structure could impair the sperm aster formation still needs to be clarified.

\section{IMSI outcome}

Sperm selection at a high microscope magnification highlighted the need to evaluate sperm morphology to assess male fertility potential and to predict ICSI outcome. Many of the published studies regarding IMSI (Table 1) rely on retrospective analyses. ${ }^{18,29,48-51}$ In these studies, the use of IMSI leads to positive results, especially in couples with severe male factor infertility or repeated ICSI failures. Specifically, IMSI achieves higher clinical pregnancy rates and lower abortion rates when compared to ICSI.

To date, only one prospective study has clearly shown a beneficial effect of IMSI on pregnancy rates. ${ }^{34}$ The primary aim of this work was to assess the potential advantages of IMSI in patients affected by severe oligoasthenoteratozoospermia (OAT) without considering previous failed ICSI attempts. A total of 219 couples underwent the ICSI procedure and 227 underwent IMSI. The main finding of the study was that IMSI granted a higher pregnancy rate. In a further analysis, the patients' population was divided into several subgroups depending on the number of previously failed ICSI attempts, to evaluate any potential benefit of using IMSI. Patients with an initial poor reproductive prognosis (two or more failed ICSI attempts) significantly benefited from the treatment in terms of pregnancy rate $(29.8 \% v s .12 .9 \%)$ and miscarriage rate $(17.4 \% v s .37 .5 \%)$, confirming the data reported in other works.

Other studies do not show any statistically significant improvements in the clinical outcome. Oliveira et al. ${ }^{52}$ reported a non-significant difference in fertilisation, implantation or pregnancy rates between the two procedures, although a trend towards a lower miscarriage rate and a better ongoing pregnancy and live birth rate was observed in the IMSI group. 
Balaban et al..$^{53}$ recently analysed the clinical differences between the IMSI and ICSI procedures in an unselected population. In this prospective randomized study, higher implantation $(28.9 \% v s$. $19.5 \%)$, clinical pregnancy $(54.0 \%$ vs. $44.4 \%)$ and live-birth rates $(43.7 \%$ vs. $38.3 \%)$ were observed in the IMSI group, although these differences did not reach statistical significance. As shown by this work, the use of IMSI in patients affected by severe male factor infertility allows significantly higher implantation rates when compared to ICSI.

The results reported in a recent study by Marci et al. ${ }^{54}$ are in accordance with the Balaban et al.'s study, where no significant difference between IMSI and ICSI groups in terms of implantation, fertilisation and pregnancy rates were observed. In the IMSI group, both fertilisation $(80 \%$ vs. $77.27 \%)$ and live birth rates $(13.72 \%$ vs. $11.39 \%)$ were slightly higher than the ICSI groups, and the miscarriage rate $(5.26 \%$ vs. $17.78 \%)$ tended to be lower when compared to the ICSI group, although no significant difference was found. The IMSI procedure did not provide any benefit either in cases of male factor infertility or in couples with more than one previous ICSI failures. However, the use of IMSI in the male factor infertility subgroup was associated with a trend towards better laboratory and clinical outcomes.

In an extensive, randomized prospective study, Setti et al. ${ }^{55}$ reported similar clinical outcomes in couples undergoing either IMSI or ICSI, except that a significantly higher fertilisation rate was observed in the former procedure. A further analysis was specifically conducted on the patients with OAT, who were divided into two subgroups according to the criteria used for the diagnosis (WHO reference values of 2010 or WHO reference values of 1999). IMSI increased the fertilisation rate in both subgroups and was associated with higher implantation and pregnancy rates selectively in the OAT2010 subgroup. These findings highlight the inadequacy of the new reference values for the detection of patients with a high degree of sperm dysfunction. Therefore, patients with OAT selected in accordance with the previous reference limits (which provide too high threshold values for sperm morphology, motility and concentration) do not significantly benefit from IMSI treatment when compared to conventional ICSI.

Berkovitz et al. ${ }^{56}$ confirmed that the selection and the subsequent injection of spermatozoa with normal nuclear morphology reduced the risk of major foetal malformations. A total of 185 foetuses were analysed for each IMSI and ICSI group. A significantly reduced risk of major congenital malformations was recorded for the IMSI group (2.8 vs. 7.9) despite similar rates of late spontaneous abortions and terminated pregnancies due to foetal malformations and perinatal death.

A recent study ${ }^{57}$ evaluated the influence of IMSI on the gender of the embryos. The study population was divided into 80 ICSI- and 80 IMSI-treated couples. All embryos were biopsied and the obtained blastomeres were examined using fluorescent in situ hybridisation. The results obtained from sex chromosomal analysis showed a significantly higher incidence of female embryos obtained after IMSI than after ICSI ( $65.1 \%$ vs. 54\%). The authors proposed two possible interesting explanations for this result:

- The structure of the Y chromosome is often altered owing to microdeletions, polymorphisms and genome instability. Such pathological events, in association with sperm antioxidants and DNA-repair system deficiencies, are responsible for nuclear morphological alterations that exclude the Y-bearing sperm during the selection phase at high magnification. 
- The currently applied selection criteria themselves, and in particular some unknown morphological features, may better characterize the Y-bearing sperm chromosome.

In a subsequent letter to the editor, Zech et al. ${ }^{58}$ reported data from three infertility centres in Austria and France, demonstrating that the gender distribution of babies born after IMSI did not correspond to the findings of Setti et al. In fact, considering 1852 babies born after IMSI, $51.2 \%$ were female and $48.8 \%$ were male. The same authors assumed that several factors influenced the gender ratio in the study of Setti et al. and that IMSI alone should not be considered as a critical factor for gender selection. ${ }^{58}$

\section{CONCLUSIONS}

The IMSI procedure has challenged the traditional perception of the normal appearance for a spermatozoon judged to be suitable for fertilisation injection. A spermatozoon classified as 'normal' by a low power microscopic observation may still contain ultra-structural defects. However, like ICSI, the selection of motile sperm with normal morphology (normal head without vacuoles, normal midpiece without cytoplasmic droplets and aligned with the head main axis, normal tail clearly composed of a single axoneme) remains a critical step, which requires a skilled operator and may take several minutes per spermatozoon. Unfortunately, the microscopic criteria currently used for the morphologic assessment are subjective and currently not sufficiently accurate.

IMSI could be considered a useful tool for the following clinical conditions:

- high rate of sperm aneuploidy;

- high levels of DNA fragmentation;

- repeated ICSI failures;

- marked alterations of seminal parameters due to severe testiculopathy.

Currently, standardized clinical indications are lacking. Further prospective randomized studies are required to confirm the advantages of using IMSI for specific groups of patients. To date, IMSI should be considered as a routinely used assisted reproductive technology technique due to the lack of either a standardized method or a worldwide accepted patient selection criteria.

\section{AUTHOR CONTRIBUTIONS}

GLM and IP substantially contributed to the design, preparation, drafting and revising of the final version of the manuscript and provided significant intellectual support. FM, MG and RM substantially contributed to the preparation, drafting and revising of the final version of the manuscript and RM also provided extremely important intellectual support. All authors read and approved the final version of the manuscript.

\section{COMPETING FINANCIAL INTERESTS}

The authors declare no competing financial interests.

1 http://www.asrm.org/topics/detail.aspx?id=1331

2 Bartoov B, Eltes F, Pansky M, Langzam J, Reichart M et al. Improved diagnosis of male fertility potential via a combination of quantitative ultramorphology and routine semen analyses. Hum Reprod 1994; 9: 2069-75.

3 Bartoov B, Eltes F, Pansky M, Lederman H, Caspi E et al. Estimating fertility potential via semen analysis data. Hum Reprod 1993; 8: 65-70.
4 Berkovitz A, Eltes F, Soffer Y, Zabludovsky N, Beyth Y et al. ART success and in vivo sperm cell selection depend on the ultramorphological status of spermatozoa. Andrologia 1999; 31: 1-8.

5 Kruger TF, Acosta AA, Simmons KF, Swanson RJ, Matta JF et al. Predictive value of sperm morphology in in vitro fertilization. Fertil Steril 1988; 49: 112-7.

6 Kahraman S, Akarsu C, Cengiz G, Dirican K, Sözen E et al. Fertility of ejaculated and testicular megalohead spermatozoa with intracytoplasmic sperm injection. Hum Reprod 1999; 14: 726-30.

7 Osawa $\mathrm{Y}$, Sueoka K, Iwata S, Shinohara M, Kobayashi $\mathrm{N}$ et al. Assessment of the dominant abnormal form is useful forpredicting the outcome of intracytoplasmic sperm injection in the case of severe teratozoospermia. J Assist Reprod Genet 1999; 16: 436-42.

8 Lundin K, Söderlund B, Hamberger L. The relationship between sperm morphology and rates of fertilization, pregnancy and spontaneous abortion in an in-vitro fertilization/intracytoplasmic sperm injection programme. Hum Reprod 1997; 12: 2676-81.

9 De Vos A, Van De Velde H, Joris H, Verheyen G, Devroey P et al. Influence of individual sperm morphology on fertilisation, embryo morphology, and pregnancy outcome of intracytoplasmic sperm injection. Fertil Steril 2003; 79: 42-8.

10 Miller JE, Smith TT. The effect of intracytoplasmic sperm injection and semen parameters on blastocyst development in vitro. Hum Reprod 2001; 16: 918-24.

11 Irvine DS, Twigg JP, Gordon EL, Fulton N, Milne PA et al. DNA integrity in human spermatozoa: relationships with semen quality. J Androl 2000; 21: 33-44.

12 Zini A, Libman J. Sperm DNA damage: clinical significance in the era of assisted reproduction. CMAJ 2006; 175: 495-500.

13 Loutradi KE, Tarlatzis BC, Goulis DG, Zepiridis L, Pagou T et al. The effects of sperm quality on embryo development after intracytoplasmic sperm injection. J Assist Reprod Genet 2006; 23: 69-74.

14 Bonduelle M, Camus M, De Vos A, Staessen C, Tournaye H et al. Seven years of intracytoplasmic sperm injection and follow-up of 1987 subsequent children. Hum Reprod 1999; 1: 243-64.

15 Sakkas D, Urner F, Bizzaro D, Manicardi G, Bianchi PG et al. Sperm nuclear DNA damage and altered chromatin structure: effect on fertilization and embryo development. Hum Reprod 1998; 13(Suppl4): 11-9.

16 Tomlinson MJ, Moffatt O, Manicardi GC, Bizzaro D, Afnan M et al. Interrelationship between seminal parameters and sperm nuclear DNA damage before and after density gradient centrifugation: implications for assisted conception. Hum Reprod 2001; 16: 2160-5.

17 Bartoov B, Berkovitz A, Eltes F, Kogosowski A, Menezo Y et al. Real-time fine morphology of motile human sperm cells is associated with IVF-ICSI outcome. $J$ Androl 2002; 23: 1-8.

18 Bartoov B, Berkovitz A, Eltes F, Kogosovsky A, Yagoda A et al. Pregnancy rates are higher with intracytoplasmic morphologically selected sperm injection than with conventional intracytoplasmic injection. Fertil Steril 2003; 80: 1413-9.

19 Nagy ZP, Verheyen G, Tournaye H, Van Steirteghem AC. Special applications of intracytoplasmic sperm injection: the influence of sperm count, motility, morphology, source and sperm antibody on the outcome of ICSI. Hum Reprod 1998; 13 Suppl 1: 143-54.

20 Tomlinson MJ, Moffatt O, Manicardi GC, Bizzaro D, Afnan M et al. Interrelationship between seminal parameters and sperm nuclear DNA damage before and after density gradient centrifugation: implications for assisted conception. Hum Reprod 2001; 16: 2160-5.

21 Bosco L, Ruvolo G, Morici G, Manno M, Cittadini E et al. Apoptosis in human unfertilized oocytes after intracytoplasmic sperm injection. Fertil Steril 2005; 84: 1417-23.

22 Razavi S, Nasr-Esfahani MH, Mardani M, Mafi A, Moghdam A. Effect of human sperm chromatin anomalies on fertilization outcome post-ICSI. Andrologia 2003; 35: 23843.

23 Larson KL, DeJonge CJ, Barnes AM, Jost LK, Evenson DP. Sperm chromatin structure assay parameters as predictors of failed pregnancy following assisted reproductive techniques. Hum Reprod 2000; 15: 1717-22.

24 Halliday J. Outcomes for offspring of men having ICSI for male factor infertility. Asian J Androl 2012; 14: 116-20.

25 Bonduelle M, Wennerholm UB, Loft A, Tarlatzis BC, Peters C et al. A multicentre cohort study of the physical health of 5 year old children conceived after intracytoplasmic sperm injection, in vitro fertilization and natural conception. Hum Reprod 2005; 20: 413-9.

26 Silber SJ, Repping S. Transmission of male infertility to future generations: lessons from the Y chromosome. Hum Reprod Update 2002; 8: 217-29.

27 Bartoov B, Eltes F, Weissenberg R, Lunenfeld B. Morphological characterization of abnormal human spermatozoa using transmission electron microscopy. Arch Androl 1980; 5: 305-22.

28 Bartoov B, Eltes F, Reichart M, Langzam J, Lederman $\mathrm{H}$ et al. Quantitative ultramorphological analysis of human sperm: fifteen years of experience in the diagnosis and management of male factor infertility. Arch Androl 1999; 43: 13-25.

29 Hazout A, Dumont-Hassan M, Junca AM, Cohen Bacrie P, Tesarik J. Highmagnification ICSI overcomes paternal effect resistant to conventional ICSI. Reprod Biomed Online 2006; 12: 19-25.

30 Antinori M, Licata E, Dani G, Cerusico F, Versaci C et al. Intracytoplasmic morphologically selected sperm injection: a prospective randomized trial. Reprod Biomed Online 2008; 16: 835-41.

31 Zini A, Libman J. Sperm DNA damage: clinical significance in the era of assisted reproduction. CMAJ 2006; 175: 495-500. 
32 Khadem N, Poorhoseyni A, Jalali M, Akbary A, Heydari ST. Sperm DNA fragmentation in couples with unexplained recurrent spontaneous abortions. Andrologia; 2012 Dec 20. doi: 10.1111/and.12056. [Epub ahead of print].

33 Hammoud I, Boitrelle F, Ferfouri F, Vialard F, Bergere M et al. Selection of norma spermatozoa with a vacuole-free head (x6300) improves selection of spermatozo with intact DNA in patients with high sperm DNA fragmentation rates. Andrologia 2013; 45: 163-70.

34 Chelli MH, Albert M, Ray PF, Guthauser B, Izard V et al. Can intracytoplasmic morphologically selected sperm injection be used to select normal-sized sperm heads in infertile patients with macrocephalic sperm head syndrome? Fertil Steril 2010; 93: 1347.e1-5.

35 Kacem O, Sifer C, Barraud-Lange V, Ducot B, De Ziegler D et al. Sperm nuclear vacuoles, as assessed by motile sperm organellar morphological examination, are mostly of acrosomal origin. Reprod Biomed Online 2010; 20: 132-7.

36 Roldan ERS. Better intracytoplasmic sperm injection without sperm membranes and acrosome. Proc Natl Acad Sci USA 2006; 103: 17585-6.

37 Peer S, Eltes F, Berkovitz A, Yehuda R, Itsykson P et al. Is fine morphology of the human sperm nuclei affected by in vitro incubation at 37 degrees C? Fertil Steril 2007: 88: 1589-94.

38 Shi Q, Martin RH. Aneuploidy in human spermatozoa: FISH analysis in men with constitutional chromosomal abnormalities, and in infertile men. Reproduction 2001; 121: 655-66.

39 Shi $\mathrm{Q}$, Martin $\mathrm{RH}$. Aneuploidy in human sperm: a review of the frequency and distribution of aneuploidy, effects of donor age and lifestyle factors. Cytogenet Cell Genet 2000; 90: 219-26.

40 Figueira Rde C, Braga DP, Setti AS, laconelli A Jr, Borges E Jr. Morphological nuclear integrity of sperm cells is associated with preimplantation genetic aneuploidy screening cycle outcomes. Fertil Steril 2011; 95: 990-3.

41 Vanderzwalmen P, Hiemer A, Rubner P, Bach M, Neyer A et al. Blastocyst development after sperm selection at high magnification is associated with size and number of nuclear vacuoles. Reprod Biomed Online 2008; 17: 617-27.

42 Cassuto NG, Bouret D, Plouchart JM, Jellad S, Vanderzwalmen P et al. A new real-time morphology classification for human spermatozoa: a link for fertilization and improved embryo quality. Fertil Steril 2009; 92: 1616-25.

43 Knez K, Zorn B, Tomazevic T, Vrtacnik-Bokal E, Virant-Klun I. The IMSI procedure improves poor embryo development in the same infertile couples with poor semen quality: a comparative prospective randomized study. Reprod Biol Endocrinol 2011 9:123 doi:10.1186/1477-7827-9-123.

44 Knez K, Tomazevic T, Zorn B, Vrtacnik-Bokal E, Virant-Klun I. Intracytoplasmic morphologically selected sperm injection improves development and quality of preimplantation embryos in teratozoospermia patients. Reprod Biomed Online 2012; 25: 168-79.

45 Tesarik J. Paternal effects on cell division in the human preimplantation embryo. Reprod Biomed Online 2005; 10:370-5.
46 Mauri AL, Petersen CG, Oliveira JB, Massaro FC, Baruffi RL et al. Comparison of day 2 embryo quality after conventional ICSI versus intracytoplasmic morphologically selected sperm injection (IMSI) using sibling oocytes. Eur J Obstet Gynecol Reprod Biol 2010; 150: 42-6.

47 Ugajin T, Terada Y, Hasegawa H, Nabeshima H, Suzuki K et al. The shape of the sperm midpiece in intracytoplasmic morphologically selected sperm injection relates sperm centrosomal function. J Assist Reprod Genet 2010; 27: 75-81.

48 Bartoov B, Berkovitz A, Eltes F. Selection of spermatozoa with normal nuclei to improve the pregnancy rate with intracytoplasmic sperm injection. N Engl J Med 2001; 345: 1067-8.

49 Berkovitz A, Eltes F, Ellenbogen A, Peer S, Feldberg D et al. Does the presence of nuclear vacuoles in human sperm selected for ICSI affect pregnancy outcome? Hum Reprod 2006; 21 : 1787-90.

50 Berkovitz A, Eltes F, Lederman H, Peer S, Ellenbogen A et al. How to improve IVF-ICS outcome by sperm selection. Reprod Biomed Online 2006; 12: 634-8.

51 Berkovitz A, Eltes F, Yaari S, Katz N, Barr I et al. The morphological normalcy of the sperm nucleus and pregnancy rate of intracytoplasmic injection with morphologically selected sperm. Hum Reprod 2005; 20: 185-90.

52 Oliveira JB, Cavagna M, Petersen CG, Mauri AL, Massaro FC et al. Pregnancy outcomes in women with repeated implantation failures after intracytoplasmic morphologically selected sperm injection (IMSI). Reprod Biol Endocrinol 2011; 9:99 doi:10.1186/1477-7827-9-99.

53 Balaban B, Yakin K, Alatas C, Oktem O, Isiklar A et al. Clinical outcome of intracytoplasmic injection of spermatozoa morphologically selected under high magnification: a prospective randomized study. Reprod Biomed Online. $2011 \mathrm{Feb}$ 15. [Epub ahead of print].

54 Marci R, Murisier F, Lo Monte G, Soave I, Chanson A et al. Clinical outcome after IMS procedure in an unselected infertile population: a pilot study. Reprod Health 2013 10:16 doi: 10.1186/1742-4755-10-16.

55 Setti AS, Figueira Rde C, Braga DP, laconelli A Jr, Borges E Jr. Intracytoplasmic morphologically selected sperm injection benefits for patients with oligoasthenozoospermia according to the 2010 World Health Organization reference values. Fertil Steril 2011; 95: 2711-4.

56 Berkovitz A, Eltes F, Paul M, Adrian E, Benjamin B. The chance of having a healthy normal child following intracytoplasmic morphologically-selected sperm injection (IMSI) treatment is higher compared to conventional IVF-ICSI treatment. Fertil Steril 2007; 88: S20.

57 Setti AS, Figueira RC, Braga DP, laconelli A Jr, Borges E Jr. Gender incidence of intracytoplasmic morphologically selected sperm injection-derived embryos: a prospective randomized study. Reprod Biomed Online 2012; 24: 420-3.

58 Zech NH, Wirleitner B, Cassuto NG, Schuff M, Spitzer D et al. Re: Gender incidence of intracytoplasmic morphologically selected sperm injection-derived embryos: a prospective randomized study. Reprod Biomed Online 2012; 25: 333. 